\title{
Article \\ GmNF-YC4-2 Increases Protein, Exhibits Broad Disease Resistance and Expedites Maturity in Soybean
}

\author{
Seth $\mathrm{O}^{\prime}$ Conner ${ }^{1}$, Wenguang Zheng ${ }^{2}$, Mingsheng $\mathrm{Qi}^{3}{ }^{3}{ }^{\mathbb{O}}$, Yuba Kandel $^{3}$, Robert Fuller ${ }^{1}$, Steven A. Whitham ${ }^{3}$ \\ and Ling $\mathrm{Li}^{1, * \mathbb{C}}$ \\ 1 Department of Biological Sciences, Mississippi State University, Mississippi State, MS 39762, USA; \\ sjo88@msstate.edu (S.O.); robmfuller2@gmail.com (R.F.) \\ 2 Department of Genetics, Development and Cell Biology, Iowa State University, Ames, IA 50011, USA; \\ wgzheng2008@gmail.com \\ 3 Department of Plant Pathology and Microbiology, Iowa State University, Ames, IA 50011, USA; \\ tonyqms@gmail.com (M.Q.); ykandel@iastate.edu (Y.K.); swhitham@iastate.edu (S.A.W.) \\ * Correspondence: liling@biology.msstate.edu
}

check for

updates

Citation: O'Conner, S.; Zheng, W.; Qi, M.; Kandel, Y.; Fuller, R.;

Whitham, S.A.; Li, L. GmNF-YC4-2 Increases Protein, Exhibits Broad Disease Resistance and Expedites Maturity in Soybean. Int. J. Mol. Sci. 2021, 22, 3586. https://doi.org/ $10.3390 /$ ijms22073586

Academic Editor: Bartolome Sabater

Received: 5 March 2021

Accepted: 26 March 2021

Published: 30 March 2021

Publisher's Note: MDPI stays neutral with regard to jurisdictional claims in published maps and institutional affiliations.

Copyright: (c) 2021 by the authors. Licensee MDPI, Basel, Switzerland. This article is an open access article distributed under the terms and conditions of the Creative Commons Attribution (CC BY) license (https:// creativecommons.org/licenses/by/ $4.0 /)$.

\begin{abstract}
The NF-Y gene family is a highly conserved set of transcription factors. The functional transcription factor complex is made up of a trimer between NF-YA, NF-YB, and NF-YC proteins. While mammals typically have one gene for each subunit, plants often have multigene families for each subunit which contributes to a wide variety of combinations and functions. Soybean plants with an overexpression of a particular NF-YC isoform GmNF-YC4-2 (Glyma.04g196200) in soybean cultivar Williams 82, had a lower amount of starch in its leaves, a higher amount of protein in its seeds, and increased broad disease resistance for bacterial, viral, and fungal infections in the field, similar to the effects of overexpression of its isoform GmNF-YC4-1 (Glyma.06g169600). Interestingly, GmNF-YC4-2-OE (overexpression) plants also filled pods and senesced earlier, a novel trait not found in GmNF-YC4-1-OE plants. No yield difference was observed in GmNF-YC4-2-OE compared with the wild-type control. Sequence alignment of GmNF-YC4-2, GmNF-YC4-1 and AtNF-YC1 indicated that faster maturation may be a result of minor sequence differences in the terminal ends of the protein compared to the closely related isoforms.
\end{abstract}

Keywords: NF-YC4 transcription factor; early maturation; disease resistance; seed protein

\section{Introduction}

Effectively feeding an ever-growing global population begins with nutrient rich, resilient crops. Over one in seven people in the world do not have access to a sufficient protein supply in their diets [1]. In addition, the majority of the population relies on a plant-based diet for their protein uptake [2]. As most livestock also requires plant-based diets for their protein, the dietary needs of the world can mostly be attributed to plants. Thus, generating plants with increased protein content can help efficiently feed the global population.

Crop plants such as Glycine max (soybean) are grown in a wide range of environments and face a multitude of both biotic and abiotic challenges. While plants have evolved a complex immune system [3-7], crops across the world suffer yield loss due to diseases [8-11] and environmental factors [12,13]. Many genetic engineering methods have been used to combat these factors $[8,14]$. However, many of these induce constitutively active defense responses that can in turn negatively affect the growth and yield of the plant $[15,16]$. For example, silencing mitogen-activated protein kinase MAPK4 in soybean plants severely stunts the plants when providing a resistance to pathogens [17]. Therefore, genetic engineered resistance that allows for an enhanced pathogen resistance while avoiding major growth defects is important. 
While plants have evolved a complex immune system to deal with minor biotic and abiotic perturbations, they have also evolved a more drastic mechanism to deal with seasonal environmental changes. As plants are sessile organisms, rather than maintaining full function throughout a harsh season, they instead undergo programmed cell death called senescence [18-20]. This process allows for nutrient re-localization, such as nitrogen remobilization from leaves to seeds, and it is important for seed quality and nitrogen use efficiency [21-24]. With the looming threat of climate change and the growing global population, the challenge set before us is to improve crop nitrogen use efficiency in order to allow for less reliance on nitrate fertilizers [22]. It has been speculated that stimulating autophagy during stress response could improve crops' resistance to the effects of climate change [22].

Previous work from our lab has identified an orphan gene, Qua Quine Starch (QQS), in Arabidopsis thaliana with a role in carbon and nitrogen allocation and disease resistance: QQS increased leaf and seed protein content, and increased plant resistance to pathogens, bacterium, virus, fungus and pests without impacting the plant growth and yield [25-28]; Nuclear Factor Y subunit C4 (NF-YC4) was its interacting partner [25-28] and had similar functions as QQS. Further work showed that homologs of NF-YC4 in crop species confer similar effects [25,26]. In soybean, GmNF-YC4-1-OE (overexpression) plants demonstrate increased protein content and broad disease resistance, similar to the effect of AtNFYC4-OE Arabidopsis plants [25-27,29]. Here, we show that another NF-YC4 isoform in soybean GmNF-YC4-2, when overexpressed, has increased protein content and broad disease resistance with no significant effect on seed yield. Unlike GmNF-YC4-1, GmNF-YC42 has a role in plant maturity and senescence-faster pod filling and earlier senescence. This enhanced role of GmNF-YC4-2 may likely be the result of small changes in the gene sequence near the terminal ends. Our results demonstrate that overexpressing GmNF-YC42 increased carbon and nitrogen allocation to protein, increased broad disease resistance, and hastened plant maturation.

\section{Results}

2.1. GmNF-YC4-2-OE Plants Have High Transcript Levels of GmNF-YC4-2 in Leaves and Seeds

To confirm the efficacy of our OE vectors, the transcript level of GmNF-YC4-2 was assessed in leaves and seeds. The transcript level of GmNF-YC4-2 in leaves of GmNF-YC4-2$O E$ lines ranged from 12.3-16.4 average relative quantification (RQ) compared to wild type (WT) (Figure 1A). That magnitude was increased in seeds where the average RQ ranged from 31.0-69.3 (Figure 1A). The validity of the $35 \mathrm{~S}$ driven $\mathrm{OE}$ vectors used in this study was assessed by GUS ( $\beta$-glucuronidase) staining of 35S-QQS-GUS constructs in soybean. Moderate to high levels of GUS signal were present in leaves, flowers, pods and seeds, indicating $35 \mathrm{~S}$ activity in these tissues (Figure 1B-D) and confirming previous studies demonstrating no tissue specificity for the CaMV 35S promoter [30].

\subsection{GmNF-YC4-2 Is Involved in Regulation of Plant Composition}

Similar to previous studies in GmNF-YC4-1-OE plants [27], GmNF-YC4-2-OE plants also showed altered leaf and seed composition. GmNF-YC4-2-OE plants had a decrease in leaf starch (Figure 2A,B, $p<0.05$ for GmNF-YC4-2-OE 1,2 and $p<0.1$ for GmNF-YC4-2-OE 3 ) and leaf protein content was increased (Figure $2 \mathrm{C}, p<0.01$ for GmNF-YC4-2-OE 1 and $p<0.05$ for GmNF-YC4-2-OE 2,3). 
A

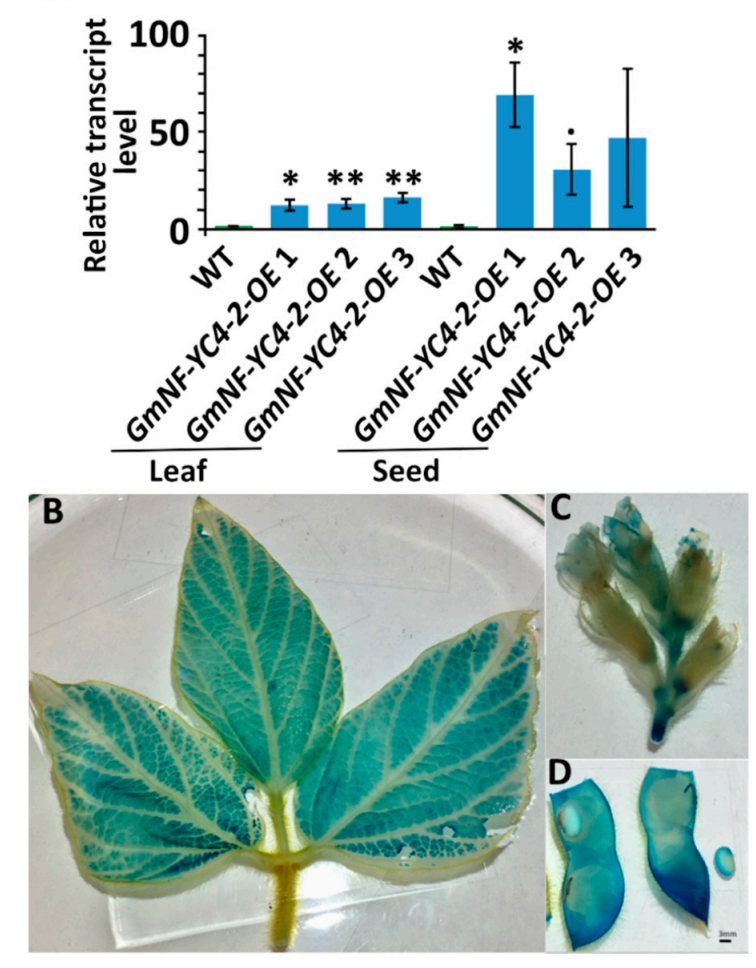

Figure 1. GmNF-YC4-2 transcript level was increased in leaf and seed tissue in GmNF-YC4-2-OE plants. (A) Quantitative reverse transcription PCR of GmNF-YC4-2 transcript level. GUS staining of 35S-QQS-GUS soybean plants shows 35S drove expression of GUS in leaves (B), flowers (C), and pods/seeds (D). All data in bar chart shows mean \pm SE (Standard Error), $n=3$. A two-tailed Student's t-test was used to compare GmNF-YC4-2-OE and WT, ${ }^{* *} p<0.01{ }^{*} p<0.05 ;{ }^{\bullet} p<0.1$.
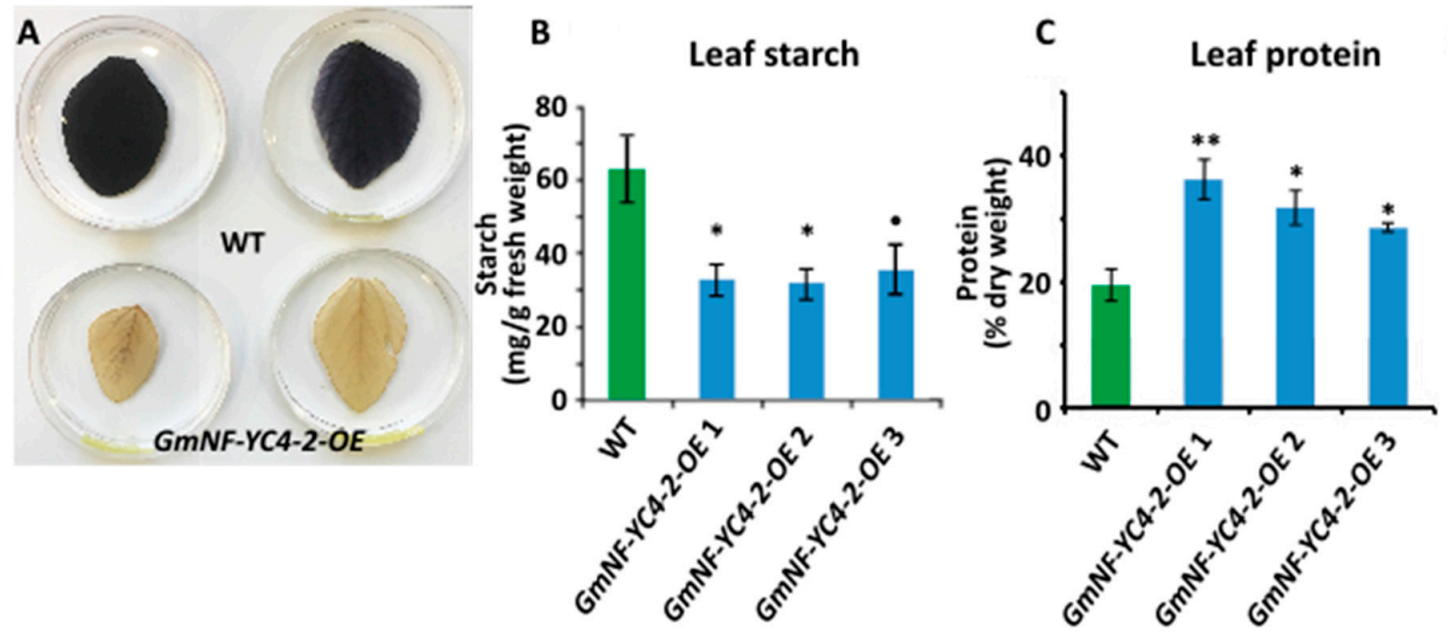

Figure 2. Leaf starch and protein composition was altered in GmNF-YC4-2-OE plants. Leaf starch content in GmNF-YC4-2$O E$ plants was decreased when compared to WT, indicated by starch staining (A) and quantification (B). (C) Leaf protein content was increased in GmNF-YC4-2-OE plants. All data in bar charts show mean $\pm \mathrm{SE}, n=3$ or 4 (in C). A two-tailed Student's t-test was used to compare GmNF-YC4-2-OE and WT, ${ }^{* *} p<0.01{ }^{*} p<0.05 ; \bullet p<0.1$.

Metabolic analysis on GmNF-YC4-2-OE seeds was performed to determine if this metabolic effect in leaf tissue was unique. Near Infrared Spectroscopy (NIRS) analysis on seeds showed a significant increase in protein levels and decreased oil levels in three independent GmNF-YC4-2-OE lines compared to WT plants (Figure 3A, $p<0.01$ for all three lines). Chemical analysis on crushed seeds was performed to test levels of ash, 
crude fat, crude fiber, crude protein, and total sugars. Of these metabolites, ash $(p<0.01$, $<0.1$, and $<0.05$, respectively), crude fat $(p<0.01$ for all), and crude fiber $(p<0.05$ for all) showed a significant decrease in OE lines, while protein $(p<0.01$ for all) and total sugar ( $p<0.05$ for GmNF-YC4-2-OE 1 and 3) levels were increased (Figure 3B). In addition, GmNF-YC4-2-OE plants had an even higher seed protein content, approximately 5-10\%, when compared to GmNF-YC4-1-OE plants (Figure S1, $p<0.01$ ). Interestingly, there was no significant difference in seed yield per plant between WT and OE plants (Figure 3C). Therefore, GmNF-YC4-2-OE plants display similar metabolic alterations in both leaf and seed tissue.
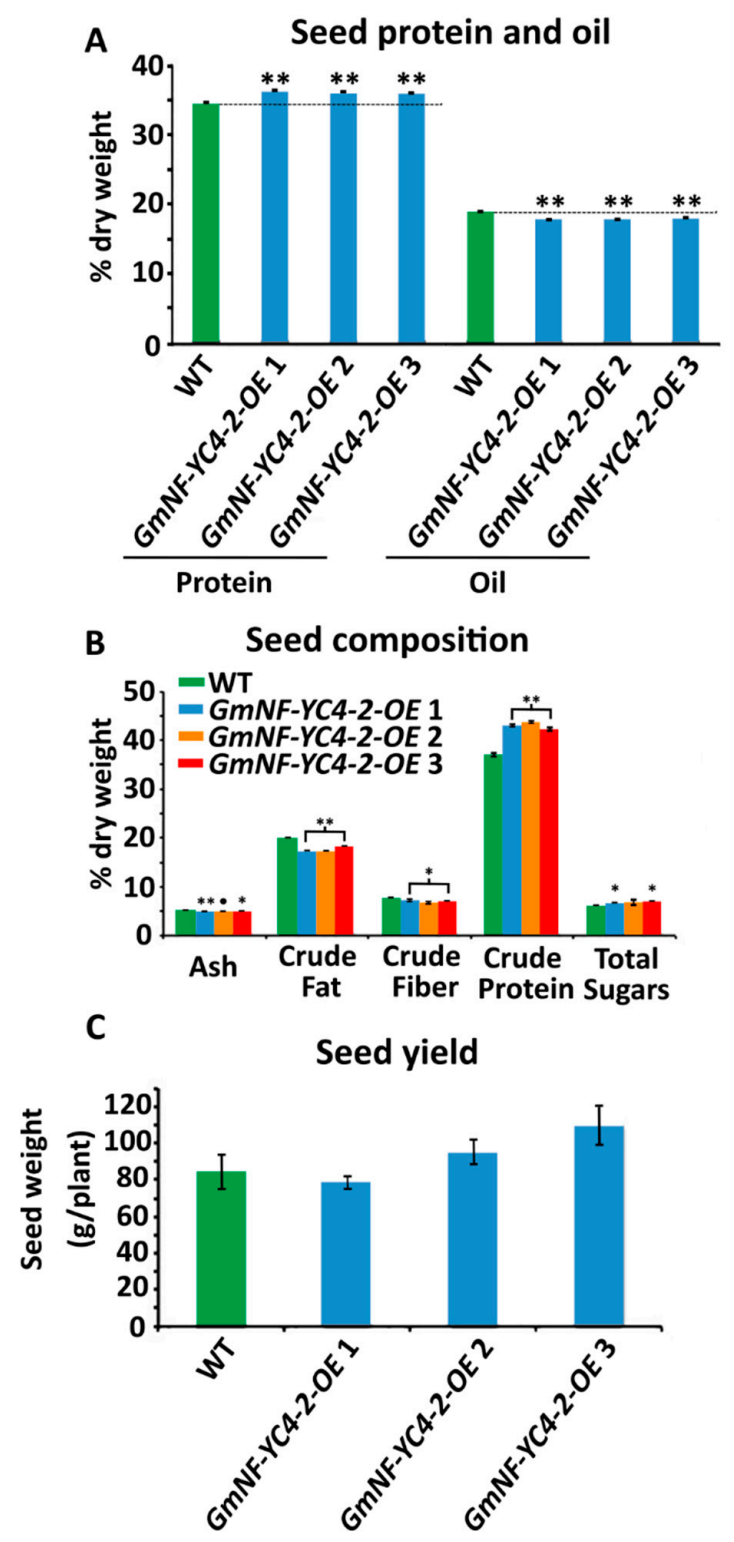

Figure 3. Seed composition was altered in GmNF-YC4-2-OE plants. (A) Seed protein content was significantly increased in all lines while oil content was significantly decreased. Composition was analyzed by near infrared spectroscopy (NIRS). (B) Ash content, crude fat and crude fiber were decreased compared to WT, while crude protein and total sugars were increased. Composition was analyzed by chemical methods. (C) No significant difference in seed yield was found. All data in bar charts show mean $\pm \mathrm{SE}$, (in A) $n=235$ (WT), 125 (GmNF-YC4-2-OE 1), 155 (GmNF-YC4-2-OE 2), 81 (GmNF-YC4-2-OE 3), (in B) $n=3$, (in C) $n=20$ (WT), 24 (GmNF-YC4-2-OE 1), 17 (GmNF-YC4-2-OE 2), and 26 (GmNF-YC4-2-OE 3). A two-tailed Student's t-test was used to compare GmNF-YC4-2-OE and $\mathrm{WT} ;{ }^{* *} p<0.01{ }^{*} p<0.05, \bullet p<0.1$. 


\subsection{GmNF-YC4-2 Confers Broad Disease Resistance}

To test if GmNF-YC4-2-OE could affect interactions between soybean and pathogens, $\mathrm{WT}$ and transgenic plants were inoculated with a virus, bacterium, and fungus. GmNFYC4-2-OE plants inoculated with a recombinant Bean pod mottle virus (BPMV) expressing green fluorescent protein (GFP) had reduced numbers of green fluorescent infection foci when compared to WT plants at both 11 and 13 days post inoculation (DPI) (Figure 4A, $p<0.01)$. The GmNF-YC4-2-OE plants were also infected with Pseudomonas syringae pv. glycinea Race 4 (PsgR4), the cause of bacterial blight. The growth of PsgR4 was decreased by 96.3\%, 96.8\%, and 92.8\% in GmNF-YC4-2-OE 1, GmNF-YC4-2-OE 2, and GmNF-YC4-2-OE 3 plants compared to Williams 82 control plants (Figure 4B, all $p<0.01$ ).
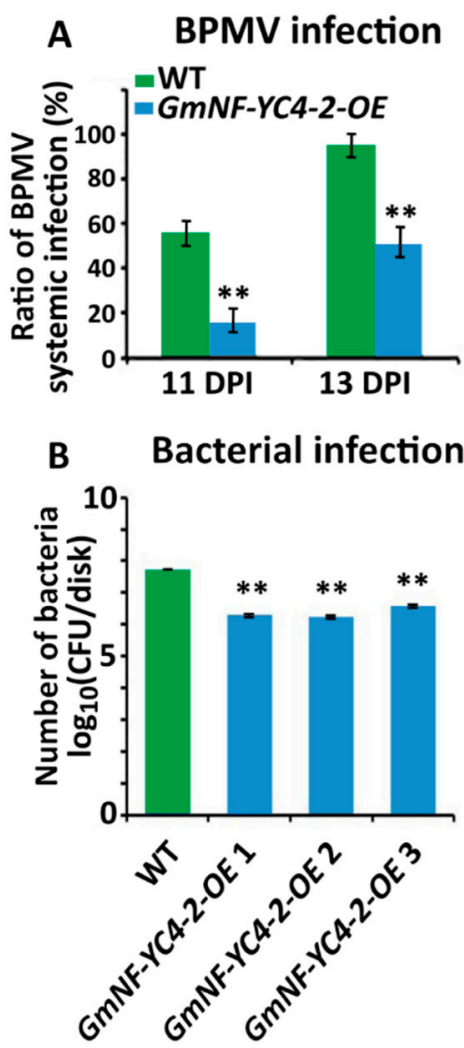

\section{SDS symptoms}

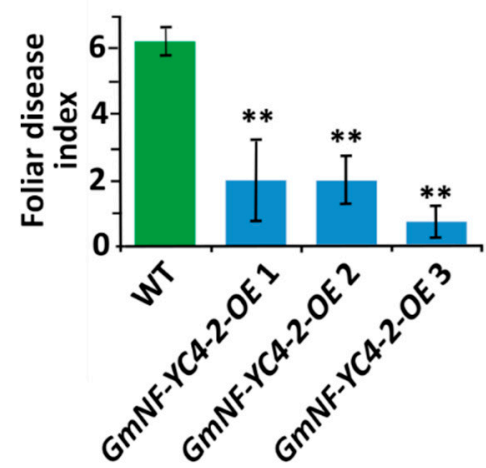

Figure 4. GmNF-YC4-2-OE plants showed enhanced disease resistance. (A) The BPMV viral foci rate was decreased at both 11 and 13 DPI in GmNF-YC4-2-OE plants compared to WT. (B) Growth of PsgR4 bacteria was reduced in GmNF-YC4-2-OE plants. CFU, colony forming units. (C) GmNF-YC4-2-OE plants also showed enhanced resistance to SDS. All data in bar charts show mean $\pm \mathrm{SE}$, (in $\mathbf{A}$ and $\mathbf{B}$ ) $n=3$, (in C) $n=5$ (WT, GmNF-YC4-2-OE 1,2), 6 (GmNF-YC4-2-OE 3). A two-tailed Student's t-test was used to compare GmNF-YC4-2-OE and WT; ** $p<0.01$. 
The plants were planted in the field and inoculated with the fungus that leads to sudden death syndrome (SDS)-Fusarium virguliforme. OE plants also displayed less severe symptoms of SDS in a field inoculation trial with a $67.8 \%, 67.8 \%$, and $88.1 \%$ decrease in foliar disease index for GmNF-YC4-2-OE 1, GmNF-YC4-2-OE 2, and GmNF-YC4-2-OE 3 respectively (Figure $4 \mathrm{C}$, all $p<0.01$ ). These data show that overexpression of GmNF-YC4-2 in soybean plants confers enhanced disease resistance to the viral, bacterial, and fungal pathogens tested along with the altered leaf and seed composition.

\subsection{GmNF-YC4-2 Regulates Plant Maturation}

GmNF-YC4-2-OE plants displayed several aspects of expedited growth. In the field, GmNF-YC4-2-OE plants showed significantly faster pod development compared to the WT plants (Figure 5A). OE plants of all three lines also showed earlier senescence compared to WT (Figure 5B). As the date of flowering was similar for both WT and OE (Figure 5C), the faster maturation was seen mainly in the transition from flowering stage to pod development. GmNF-YC4-2-OE plants contained more pods than WT at 73 Days After Planting (DAP), while WT contained more flowers (Figure 5D, flowers: $p<0.01$ for GmNF$Y C 4-2-O E 2,<0.05$ for GmNF-YC4-2-OE 3; pods: $p<0.01$ GmNF-YC4-2-OE 1 and GmNF$Y C 4-2-O E 3,<0.1$ for GmNF-YC4-2-OE 2). Thus, GmNF-YC4-2-OE plants transited quickly from flowering to pod development and this likely impacted senescence independent of flowering time. OE plants fully matured around two weeks prior to WT plants.

2.5. Minor Sequence Differences in the Terminal Ends of GmNF-YC4-2 May Be Responsible for Early Maturation Phenotype

GmNF-YC4-2 plays a similar role in carbon and nitrogen allocation and increasing broad disease resistance, as seen in both AtNF-YC4 (an Arabidopsis isoform) and GmNF$Y C 4-1$, which likely comes from the high sequence similarity within the proteins. However, unlike GmNF-YC4-1, GmNF-YC4-2 causes faster maturation when overexpressed in soybeans. This novel function possibly comes from the small sequence dissimilarities near the terminal ends of the protein (Figure 6). A phylogenetic tree was estimated for multiple NF-YC family proteins in A. thaliana, G. max and Zea mays. Based on the tree, GmNF-YC4-2 is more closely related to the NF-YC1 proteins of $A$. thaliana and Z. mays than to AtNF-YC4 (Figure S2). However, GmNF-YC4-1 and GmNF-YC4-2 are also the closest homologs in soybean to AtNF-YC4. 


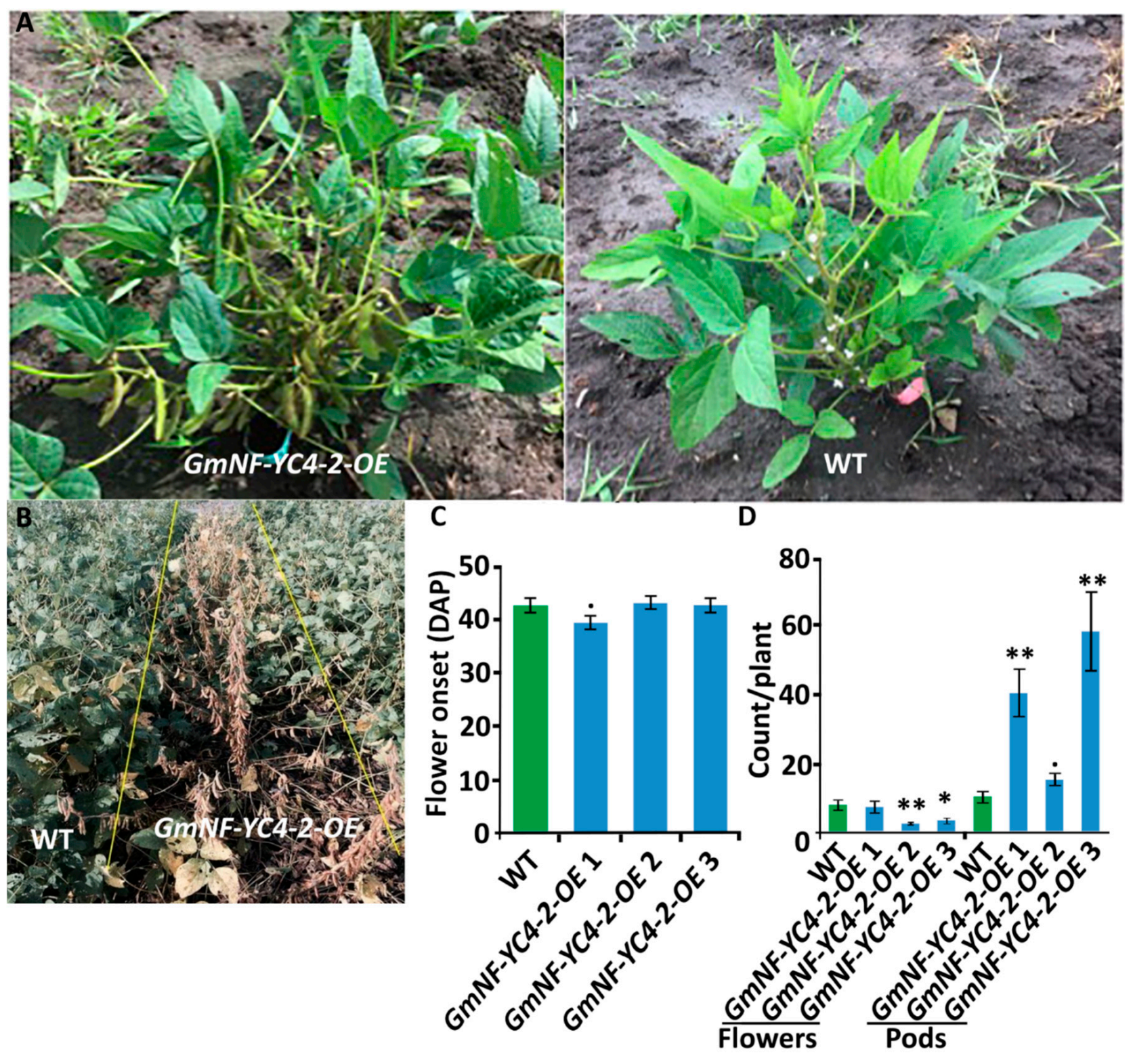

Figure 5. GmNF-YC4-2-OE plants transited from flowering stage to podding stage faster than WT plants. (A) GmNFYC4-2-OE plants showed seeding pods while WT plants were still in the flowering stage at 77 DAP. (B) GmNF-YC4-2-OE plants senesced earlier than WT plants at 96 DAP. (C) The onset of flowering (DAP of first observed open flower) was slightly faster for GmNF-YC4-2-OE 1 plants but similar in GmNF-YC4-2-OE 2 and 3 plants compared to WT. (D) At 73 DAP, pod development for GmNF-YC4-2-OE plants was advanced compared to WT plants while the number of flowers was decreased. All data in bar charts show mean $\pm \mathrm{SE}$, (in C) $n=24$ (WT), 46 (GmNF-YC4-2-OE 1), 39 (GmNF-YC4-2-OE 2), 26 (GmNF-YC4-2-OE 3); (in D) 15 (WT), 9 (GmNF-YC4-2-OE 1), 19 (GmNF-YC4-2-OE 2), 11 (GmNF-YC4-2-OE 3). A two-tailed Students t-test was used to compare GmNF-YC4-2-OE and WT; ${ }^{* *} p<0.01{ }^{*} p<0.05,{ }^{\bullet} p<0.1$. 


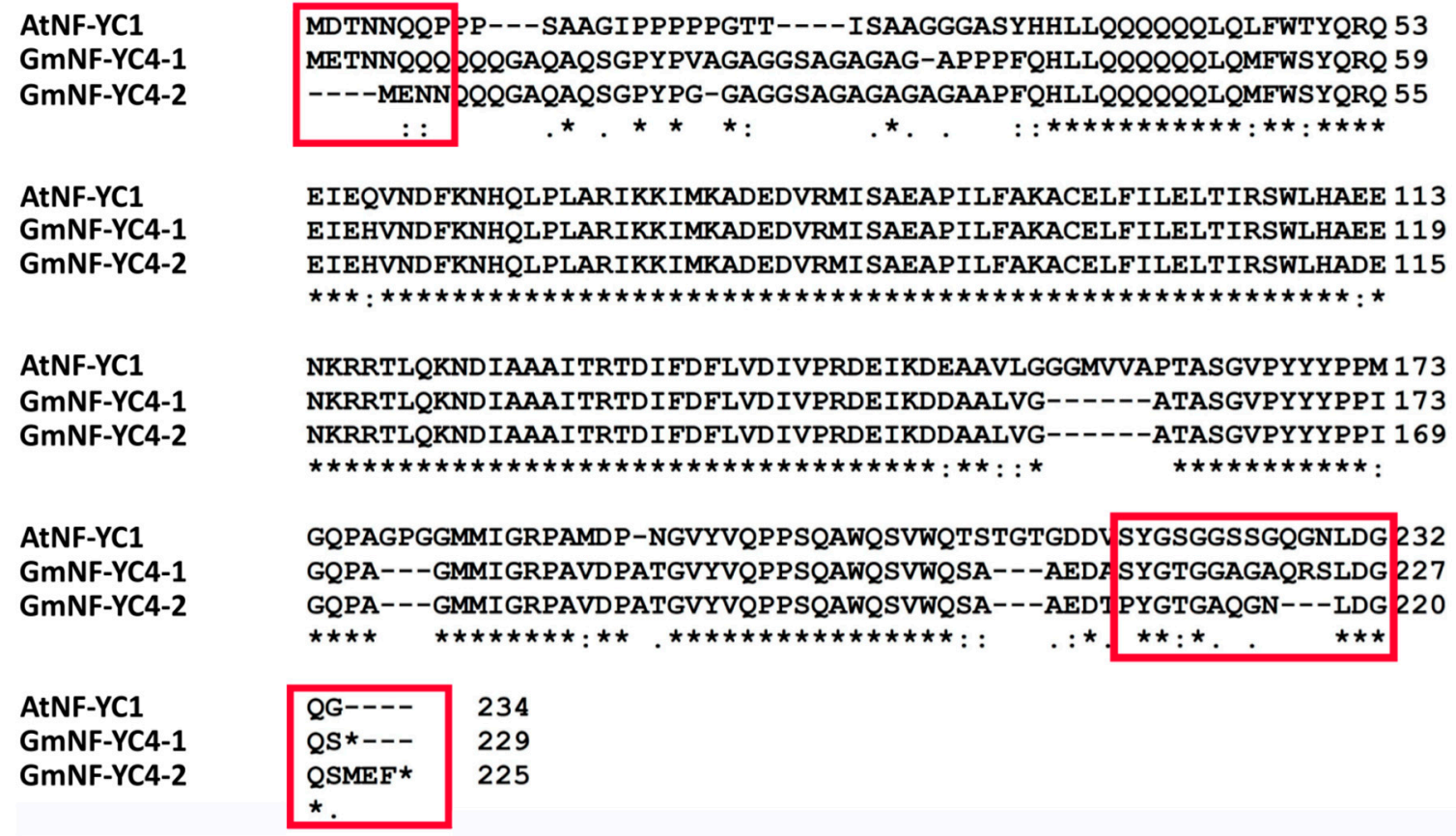

Figure 6. Protein sequence alignment of GmNF-YC4-2 with closest homolog in soybean (GmNF-YC4-1) and Arabidopsis (AtNF-YC1), neither of which has been shown to control maturation as we saw with GmNF-YC4-2. It is likely that sequence differences in the terminal ends (outlined in red) may be involved in GmNF-YC4-2's function in early maturation. The ${ }^{* \prime \prime}$ at the end of the GmNF-YC4-1 and GmNF-YC4-2 sequences signifies a stop codon.

\section{Discussion}

\subsection{GmNF-YC4-2-OE Plants Have High Transcript Levels of GmNF-YC4-2}

We checked the relative transcript levels of GmNF-YC4-2 in WT and GmNF-YC4-2$O E$ plants to determine the effectiveness of our OE vectors. The leaf tissue had a steady increase in GmNF-YC4-2 expression, while the seeds had an overall greater magnitude of expression. There was also much more variation between samples (Figure 1A). GUS staining further verified the activity of $35 \mathrm{~S}$ promoter in soybean leaves, flowers, and pods/seeds (Figure 1B-D). Together, these verified our GmNF-YC4-2-OE vectors and their effect on GmNF-YC4-2 transcript level.

\subsection{GmNF-YC4-2 and Nitrogen Metabolism and Pathogen Defense}

We previously connected the A. thaliana orphan gene Qua Quine Starch (QQS) to a role in carbon and nitrogen allocation and subsequently found the same role in its interactor AtNF-YC4 [25,28,31]. The closest NF-YC4 homologs in corn and soybean (GmNF$Y C 4-1)$ were also involved in regulating starch and protein partitioning $[25,26]$. Here, we demonstrated that another isoform of NF-YC4 in soybean, GmNF-YC4-2, is similarly involved in regulating starch and protein allocation (Figures 2 and 3). In fact, GmNF-YC4-2$O E$ seeds had higher protein content than those from GmNF-YC4-1-OE plants (Figure S1). Oil content was also decreased in GmNF-YC4-2-OE seeds (Figure 3A). This is consistent with the known inverse relationship between soybean seed protein and seed oil [32]. GmNF$Y C 4-2-O E$ plants also demonstrated a similar phenotype to GmNF-YC4-1-OE plants [27] in response to bacterial, viral and fungal pathogens (Figure 4). These similarities are expected due to the high sequence similarity between both isoforms (Figure 6).

\subsection{GmNF-YC4-2 and Plant Maturity}

While the effect on metabolism and plant defense in GmNF-YC4-2-OE plants is similar to its isoform (GmNF-YC4-1-OE plants), a faster maturity phenotype was observed in GmNF-YC4-2-OE plants. In Arabidopsis, three NF-YC transcription factors-NF-YC3, 4, and 9-act in concert to regulate flower development [33]. Therefore, it is expected that 
some G. max NF-Y transcription factors may be important for plant development. GmNFYC4-2-OE plants demonstrated several aspects of faster maturity. The onset of flowering was not significantly altered (Figure $5 \mathrm{C}$ ), but the time from flowering to pod filling was hastened (Figure 5A,D). In addition, whole-plant senescence occurred earlier in GmNFYC4-2-OE plants compared to WT in all three lines (Figure 5B). Typically, an alteration in plant senescence is paired with a change in flowering time. Here, the clear alteration in senescence appeared to be unlinked to a clear alteration in flowering time. In fact, a study in A. thaliana revealed a senescence quantitative trait loci (QTL) independent of flowering time [34]. In addition, QTLs for nitrogen use efficiency mapped back to the same region as the flowering independent QTL for senescence, leading the investigators to speculate a nitrogen metabolism gene to be responsible for flowering independent senescence [34]. In soybean, GmNF-YC4-2 may be a gene that connects flowering independent senescence and nitrogen metabolism. Early maturing varieties of soybean can decrease the yield loss in late planted fields [35] and can allow for improved yields and limit crop loss in a two-crop planting system [36]. Thus, the GmNF-YC4-2-OE system may be used to improve either late-field planting or used in a two-crop system.

\subsection{GmNF-YC4-2 Sequence Analysis}

The sequences of GmNF-YC4-1 and GmNF-YC4-2 are more closely related to AtNFYC1 than AtNF-YC4 (Figure 6 and Figure S2). However, based on the high similarities of function between AtNF-YC4 and the two G. max NF-YC4 homologs, they have been named NF-YC4 genes. In addition, these are the two closest homologs to AtNF-YC4 in soybean. As GmNF-YC4-1 and GmNF-YC4-2 have highly similar protein sequences, their overlap in function is expected. The novel function of GmNF-YC4-2 in plant maturity is likely the result of small sequence differences found at the terminal ends (Figure 6). Based on this work, GmNF-YC4-2 may be a highly active NF-YC family gene in plant metabolism (Figures 2 and 3), disease resistance (Figure 4), and plant maturation (Figure 5).

\section{Materials and Methods}

\subsection{Plant Materials}

Soybean plants: the GmNF-YC4-2 (Glyma.04g196200, in soybean genome version 1: Glyma04g37291) coding sequence overexpressing soybean lines in the Williams 82 background, expressed under the control of the constitutive cauliflower mosaic virus (CaMV) 35S promoter, were generated using the same vector and approach as described in [31]. Six lines of GmNF-YC4-2-OE plants were obtained after transformation. Of these six, all of them demonstrated the maturation phenotype and three lines were selected for further analysis. Similarly, 35S-QQS-GUS constructs were also generated with the same vector to overexpress QQS CDS-GUS. Transgenic plants were identified by both herbicide painting of leaf tissue and PCR to confirm T-DNA as in [31]. T3 generation seeds/plants were used for all analyses and segregated sibling WT plants were used as the controls. Quantitative reverse transcription PCR (RT-qPCR) was performed with GmNFYC4 primers (F: 5'-CCTCCCAGGCATGGCAGTCC-3', R: 5'-CCATCAAGGCTCCGCTGG$3^{\prime}$ ) and GmActin primers for endogenous control (F: 5'-GAGCTATGAATTGCCTGATGG-3', R: $5^{\prime}$-CGTTTCATGAATTCCAGTAGC-3'). Relative expression was determined using the $2^{-\Delta \Delta C t}$ method, as previously described [27].

GUS staining was performed as previously described using leaves (61 DAP), flowers (75 DAP) and pods (87 DAP) [28].

\subsection{Disease Resistance Assays}

The BPMV-GFP inoculation assay, Pseudomonas inoculation assay, and field SDS assay were performed as described in [27]. In brief, BPMV-GFP: soybean plants were grown for 14 days and then inoculated with BPMV-GFP. GFP foci were observed at 11 and 13 DPI (Days Post Inoculation), $n=5$ with 6 plants per replicate. 
Pseudomonas inoculation assay: the first trifoliate leaf on 23-day old soybeans was spray inoculated and bacterial levels were determined at 0 DPI and 4 DPI.

Field SDS assay: three independent lines were planted in six replicates in a randomized complete block design. The plants were planted in a field with a history of SDS and SDS inoculum was added to the soybeans at planting to induce uniform disease pressure. Fields were irrigated to create more favorable environment for the SDS. At stage R5 [37], the plants were assessed for SDS symptoms. SDS Incidence was estimated based on the number of plants with foliar symptoms in each row and SDS severity was estimated in the scale of $1-9$ as previously described [38,39]. The incidence and severity were converted to a foliar disease index (FDX).

\subsection{Flowering/Seeding Time}

Soybean plants, of three lines (GmNF-YC4-2-OE 1, 2, and 3) from three independent transformation events of GmNF-YC4-2-OE and Williams 82 controls, were planted at Mississippi State University Research Farm on 03 May 2019. Beginning at the first signs of flowering, plants were visited daily to monitor the onset of flowering until flowers were present on every plant. At $73 \mathrm{DAP}$, during the transition from flowering to podding, flowers and pods were counted for each plant. $n=15$ (WT), $n=9$ (GmNF-YC4-2-OE 1), $n=19$ (GmNF-YC4-2-OE 2), $n=11$ (GmNF-YC4-2-OE 3).

\subsection{Composition Analysis}

The composition analysis was conducted as previously described $[25,28,31]$, such as $\mathrm{I}_{2} / \mathrm{KI}$ staining for starch, starch quantification, and determination of protein content, $n=3$ biological replicates.

Compositional analysis of soybean seeds (protein, oil, and fiber) was conducted with near-infrared spectroscopy (NIRS) using an Infratec 1229 whole grain analyzer. Biological replicates per independent line: GmNF-YC4-2-OE 1, $n=125$; GmNF-YC4-2-OE 2, $n=155$; GmNF-YC4-2-OE 3, $n=81 ; \mathrm{WT}, n=235$.

Forty grams of seeds from each biological replicate $(n=3)$ in each of the three lines were crushed and sent to Eurofins for further composition testing of ash, crude fat, crude fiber, protein, and total sugars.

\subsection{Seed Weight per Plant}

Seeds were harvested from field-grown soybean plants and seed weight per plant was measured. Biological replicates per independent line: GmNF-YC4-2-OE 1, $n=24$; GmNF-YC4-2-OE 2, $n=17$; GmNF-YC4-2-OE 3, $n=26$; and WT, $n=20$.

\subsection{Bioinformatics Analysis}

Sequence analysis: for protein alignment visualization, sequences were aligned with Clustal Omega (1.2.4) multiple sequence alignment [40]. The maximum likelihood tree was created using MEGAX [41] based on the JTT $+\mathrm{G}+\mathrm{F}$ model. The tree with the highest log likelihood was selected.

\subsection{Accession Numbers}

Sequence data from this article can be found under the following accession numbers in The Arabidopsis Genome Information Resource: QQS (At3g30720), NF-YC4 (At5g63470), NF-YC1 (At3g48590), and in LegumeIP: GmNF-YC4-1 (Glyma06g17780) and GmNF-YC4-2 (Glyma04g196200).

Supplementary Materials: The following are available online at https:/ / www.mdpi.com/article / 10.3390/ijms22073586/s1. Figure S1: GmNF-YC4-2-OE lines had higher seed protein content than GmNF-YC4-1-OE lines. Figure S2: A maximum likelihood tree for the NF-YC family of proteins in Arabidopsis thaliana, Glycine max, and Zea mays. 
Author Contributions: Conceptualization, L.L.; Formal analysis, S.O., S.A.W. and L.L.; Investigation, S.O., W.Z., M.Q., Y.K., R.F., S.A.W. and L.L.; Supervision, L.L.; Writing—original draft, S.O. and L.L.; Writing-review and editing, S.O., W.Z., M.Q., Y.K., R.F., S.A.W. and L.L. All authors have read and agreed to the published version of the manuscript.

Funding: This research was funded by NSF award number MCB-0951170 (to L.L. (co-PI)), Iowa Soybean Association (one to L.L., and one to S.A.W.), Mississippi State University (to L.L.), and USDA NIFA Hatch project 3808 (to S.A.W.).

Data Availability Statement: Sequences for GmNF-YC4-1 and GmNF-YC4-2 were found at https: / / www.soybase.org/ (Access date 05 March 2021) and the sequence for AtNF-YC1 was found at https:/ / www.arabidopsis.org/ (Access date 05 March 2021). All other sequences were found at https:/ / www.ncbi.nlm.nih.gov/ (Access date 05 March 2021).

Acknowledgments: We thank MSU North Farm, Keith Daniels and Eddie Stevens for field management; Lei Wang, Rezwan Tanvir and Arpana Upadhyay for help with plant care; Diane Luth and Kan Wang at ISU PTF for soybean transformation; Daren Mueller for helpful discussion on SDS field experiment on soybean; Charles Hurburgh and Glen Rippke at ISU Grain Quality Laboratory for NIRS analysis of soybean seed composition; and Eurofins for soybean seed composition analysis.

Conflicts of Interest: The authors declare no conflict of interest.

\section{References}

1. Godfray, H.C.; Beddington, J.R.; Crute, I.R.; Haddad, L.; Lawrence, D.; Muir, J.F.; Pretty, J.; Robinson, S.; Thomas, S.M.; Toulmin, C. Food security: The challenge of feeding 9 billion people. Science 2010, 327, 812-818. [CrossRef]

2. Pimentel, D.; Pimentel, M. Sustainability of meat-based and plant-based diets and the environment. Am. J. Clin. Nutr. 2003, 78, 660S-663S. [CrossRef] [PubMed]

3. Jones, J.D.G.; Dangl, J.L. The plant immune system. Nature 2006, 444, 323-329. [CrossRef]

4. Katagiri, F.; Tsuda, K. Understanding the Plant Immune System. Mol. Plant-Microbe Interact. 2010, 23, 1531-1536. [CrossRef]

5. Nishimura, M.T.; Dangl, J.L. Arabidopsis and the plant immune system. Plant J. 2010, 61, 1053-1066. [CrossRef] [PubMed]

6. Han, G.-Z. Origin and evolution of the plant immune system. New Phytol. 2019, 222, 70-83. [CrossRef] [PubMed]

7. Nobori, T.; Tsuda, K. The plant immune system in heterogeneous environments. Curr. Opin. Plant Biol. 2019, 50, 58-66. [CrossRef]

8. Wulff, B.B.H.; Horvath, D.M.; Ward, E.R. Improving immunity in crops: New tactics in an old game. Curr. Opin. Plant Biol. 2011, 14, 468-476. [CrossRef]

9. Mueller, D.S.; Wise, K.A.; Sisson, A.J.; Allen, T.W.; Bergstrom, G.C.; Bosley, D.B.; Bradley, C.A.; Broders, K.D.; Byamukama, E.; Chilvers, M.I.; et al. Corn Yield Loss Estimates Due to Diseases in the United States and Ontario, Canada from 2012 to 2015. Plant Health Prog. 2016, 17, 211-222. [CrossRef]

10. Peltier, A.J.; Bradley, C.A.; Chilvers, M.I.; Malvick, D.K.; Mueller, D.S.; Wise, K.A.; Esker, P.D. Biology, Yield loss and Control of Sclerotinia Stem Rot of Soybean. J. Integr. Pest Manag. 2012, 3, B1-B7. [CrossRef]

11. Wrather, A.; Shannon, G.; Balardin, R.; Carregal, L.; Escobar, R.; Gupta, G.K.; Ma, Z.; Morel, W.; Ploper, D.; Tenuta, A. Effect of Diseases on Soybean Yield in the Top Eight Producing Countries in 2006. Plant Health Prog. 2010, 11, 29. [CrossRef]

12. Raza, A.; Razzaq, A.; Mehmood, S.S.; Zou, X.; Zhang, X.; Lv, Y.; Xu, J. Impact of Climate Change on Crops Adaptation and Strategies to Tackle Its Outcome: A Review. Plants 2019, 8, 34. [CrossRef]

13. Adhikari, U.; Nejadhashemi, A.P.; Woznicki, S.A. Climate change and eastern Africa: A review of impact on major crops. Food Energy Secur. 2015, 4, 110-132. [CrossRef]

14. Wally, O.; Punja, Z.K. Genetic engineering for increasing fungal and bacterial disease resistance in crop plants. GM Crop. 2010, 1 , 199-206. [CrossRef]

15. Bolton, M.D. Primary Metabolism and Plant Defense-Fuel for the Fire. Mol. Plant-Microbe Interact. 2009, 22, 487-497. [CrossRef]

16. Heil, M. The Ecological Concept of Costs of Induced Systemic Resistance (ISR). Eur. J. Plant Pathol. 2001, 107, 137-146. [CrossRef]

17. Liu, J.-Z.; Horstman, H.D.; Braun, E.; Graham, M.A.; Zhang, C.; Navarre, D.; Qiu, W.-L.; Lee, Y.; Nettleton, D.; Hill, J.H.; et al. Soybean Homologs of MPK4 Negatively Regulate Defense Responses and Positively Regulate Growth and Development. Plant Physiol. 2011, 157, 1363-1378. [CrossRef]

18. Lim, P.O.; Kim, H.J.; Nam, H.G. Leaf Senescence. Annu. Rev. Plant Biol. 2007, 58, 115-136. [CrossRef] [PubMed]

19. Humbeck, K. Senescence in Plants. J. Plant Growth Regul. 2014, 33, 1-3. [CrossRef]

20. Woo, H.R.; Masclaux-Daubresse, C.; Lim, P.O. Plant senescence: How plants know when and how to die. J. Exp. Bot. 2018, 69, 715-718. [CrossRef] [PubMed]

21. Guiboileau, A.; Avila-Ospina, L.; Yoshimoto, K.; Soulay, F.; Azzopardi, M.; Marmagne, A.; Lothier, J.; Masclaux-Daubresse, C. Physiological and metabolic consequences of autophagy deficiency for the management of nitrogen and protein resources in Arabidopsis leaves depending on nitrate availability. New Phytol. 2013, 199, 683-694. [CrossRef]

22. Havé, M.; Marmagne, A.; Chardon, F.; Masclaux-Daubresse, C. Nitrogen remobilization during leaf senescence: Lessons from Arabidopsis to crops. J. Exp. Bot. 2016, 68, 2513-2529. 
23. Hörtensteiner, S.; Feller, U. Nitrogen metabolism and remobilization during senescence. J. Exp. Bot. 2002, 53, 927-937. [CrossRef] [PubMed]

24. Liu, J.; Wu, Y.H.; Yang, J.J.; Liu, Y.D.; Shen, F.F. Protein degradation and nitrogen remobilization during leaf senescence. J. Plant Biol. 2008, 51, 11-19. [CrossRef]

25. Li, L.; Zheng, W.; Zhu, Y.; Ye, H.; Tang, B.; Arendsee, Z.W.; Jones, D.; Li, R.; Ortiz, D.; Zhao, X.; et al. QQS orphan gene regulates carbon and nitrogen partitioning across species via NF-YC interactions. Proc. Natl. Acad. Sci. USA 2015, 112, 14734-14739. [CrossRef]

26. O'Conner, S.; Neudorf, A.; Zheng, W.; Qi, M.; Zhao, X.; Du, C.; Nettleton, D.; Li, L. From Arabidopsis to Crops: The Arabidopsis QQS Orphan Gene Modulates Nitrogen Allocation Across Species. In Engineering Nitrogen Utilization in Crop Plants; Shrawat, A., Zayed, A., Lightfoot, D.A., Eds.; Springer International Publishing: Cham, Switzerland, 2018; pp. 95-117.

27. Qi, M.; Zheng, W.; Zhao, X.; Hohenstein, J.D.; Kandel, Y.; O'Conner, S.; Wang, Y.; Du, C.; Nettleton, D.; MacIntosh, G.C.; et al. QQS orphan gene and its interactor NF-YC4 reduce susceptibility to pathogens and pests. Plant Biotechnol. J. 2019, 17, 252-263. [CrossRef]

28. Li, L.; Foster, C.M.; Gan, Q.; Nettleton, D.; James, M.G.; Myers, A.M.; Wurtele, E.S. Identification of the novel protein QQS as a component of the starch metabolic network in Arabidopsis leaves. Plant J. 2009, 58, 485-498. [CrossRef] [PubMed]

29. Li, L.; Wurtele, E.S. Modification of Transcriptional Repressor Binding Site in NF-YC4 Promoter for Increased Protein Content and Resistance to Stress. U.S. Patent 10640781B2, 5 May 2020.

30. Yang, N.-S.; Christou, P. Cell type specific expression of a CaMV 35S-GUS gene in transgenic soybean plants. Dev. Genet. 1990, 11, 289-293. [CrossRef]

31. Li, L.; Wurtele, E.S. The QQS orphan gene of Arabidopsis modulates carbon and nitrogen allocation in soybean. Plant Biotechnol. J. 2015, 13, 177-187. [CrossRef] [PubMed]

32. Hyten, D.L.; Pantalone, V.R.; Sams, C.; Saxton, A.; Landau-Ellis, D.; Stefaniak, T.; Schmidt, M. Seed quality QTL in a prominent soybean population. Theor. Appl. Genet. 2004, 109, 552-561. [CrossRef] [PubMed]

33. Kumimoto, R.W.; Zhang, Y.; Siefers, N.; Holt, B.F., III. NF-YC3, NF-YC4 and NF-YC9 are required for CONSTANS-mediated, photoperiod-dependent flowering in Arabidopsis thaliana. Plant J. 2010, 63, 379-391. [CrossRef]

34. Wingler, A.; Purdy, S.J.; Edwards, S.-A.; Chardon, F.; Masclaux-Daubresse, C. QTL analysis for sugar-regulated leaf senescence supports flowering-dependent and -independent senescence pathways. New Phytol. 2010, 185, 420-433. [CrossRef]

35. Egli, D.B.; Bruening, W.P. Potential of Early-Maturing Soybean Cultivars in Late Plantings. Agron. J. 2000, 92, 532-537. [CrossRef]

36. Kyei-Boahen, S.; Zhang, L. Early-Maturing Soybean in a Wheat-Soybean Double-Crop System Yield and Net Returns. Agron. J. 2006, 98, 295-301. [CrossRef]

37. Fehr, W.; Caviness, C.; Burmood, D.; Pennington, J. Stage of development descriptions for soybeans, Glycine Max (L.) Merrill 1. Crop Sci. 1971, 11, 929-931. [CrossRef]

38. Gibson, P.; Shenaut, M.; Njiti, V.; Suttner, R.; Myers, O., Jr. Soybean Varietal Response to Sudden Death Syndrome. In Proceedings of the 24th Soybean Seed Res Conf, Chicago, IL, USA, 6 December 1994; pp. 6-7.

39. Kandel, Y.R.; Bradley, C.A.; Wise, K.A.; Chilvers, M.I.; Tenuta, A.U.; Davis, V.M.; Esker, P.D.; Smith, D.L.; Licht, M.A.; Mueller, D.S. Effect of glyphosate application on sudden death syndrome of glyphosate-resistant soybean under field conditions. Plant Dis. 2015, 99, 347-354. [CrossRef] [PubMed]

40. Madeira, F.; Park, Y.M.; Lee, J.; Buso, N.; Gur, T.; Madhusoodanan, N.; Basutkar, P.; Tivey, A.R.N.; Potter, S.C.; Finn, R.D.; et al. The EMBL-EBI search and sequence analysis tools APIs in 2019. Nucleic Acids Res. 2019, 47, W636-W641. [CrossRef]

41. Kumar, S.; Stecher, G.; Li, M.; Knyaz, C.; Tamura, K. MEGA X: Molecular evolutionary genetics analysis across computing platforms. Mol. Biol. Evol. 2018, 35, 1547-1549. [CrossRef] 